\title{
Optimization of exclusive release policies for hydropower reservoir operation by using genetic algorithm
}

\begin{abstract}
The efficient utilization of hydropower resources play an important role in the economic sector of power systems, where the hydroelectric plants constitute a significant portion of the installed capacity. Determination of daily optimal hydroelectric generation scheduling is a crucial task in water resource management. By utilizing the limited water resource, the purpose of hydroelectric generation scheduling is to specify the amount of water releases from a reservoir in order to produce maximum power, while the various physical and operational constraints are satisfied. Hence, new forms of release policies namely, BSOPHP, CSOPHP, and SHPHP are proposed and tested in this research. These policies could only use in hydropower reservoir systems. Meanwhile, to determine the optimal operation of each policy, real coded genetic algorithm is applied as an optimization technique and maximizing the total power generation over the operational periods is chosen as an objective function. The developed models have been applied to the Cameron Highland hydropower system, Malaysia. The results declared that by using optimal release policies, the output of power generation is increased, while these policies also increase the stability of reservoir system. In order to compare the efficiency of these policies, some reservoir performance indices such as reliability, resilience, vulnerability, and sustainability are used. The results demonstrated that SHPHP policy had the highest performance among the tested release policies.
\end{abstract}

Keyword: Genetic algorithm; Hydropower; Optimization; Release policies; Reservoir operation; Reservoir performance indices 